\title{
Comparison of Ferrite Materials for Pulse Applications
}

\author{
John A. Dinkel and Chris C. Jensen \\ Fermi National Accelerator Laboratory \\ P.O. Box 500, Batavia, Illinois 60510
}

June 1993

Presented at the 9th IEEE Pulsed Power Conference, Albuquerque, New Mexico, June 21-23, 1993 


\section{Disclaimer}

This report was prepared as an account of work sponsored by an agency of the United States Government. Neither the United States Government nor any agency thereof, nor any of their employees, makes any warranty, express or implied, or assumes any legal liability or responsibility for the accuracy, completeness, or usefulness of any information, apparatus, product, or process disclosed, or represents that its use would not infringe privately owned rights. Reference herein to any specific commercial product, process, or service by trade name, trademark, manufacturer, or otherwise, does not necessarily constitute or imply its endorsement, recommendation, or favoring by the United States Government or any agency thereof. The views and opinions of authors expressed herein do not necessarily state or reflect those of the United States Government or any agency thereof. 
John A. Dinkel, Chris C. Jensen

Fermilab, P. O. Box 500, M/S 308

Batavia IL 60510

\section{Abstract}

Materials are the limiting factor in many pulse power projects. The magnetic materials available from several manufacturers were experimentally compared for their usefulness in high speed magnetic field applications. This particular application is a high speed kicker magnet for manipulation of a charged particle beam.

\section{Introduction}

Magnetic materials are typically used to guide magnetic energy, either for storage or for immediate use. The application which prompted this study is the manipulation of charged particle bunches with magnetic fields. For fast kicker magnets with modest magnetic fields, the response of the ferrite can be a limiting factor. Since the function of this ferrite is to efficiently guide magnetic energy into a vacuum gap, the magnetization must increase to the required value as rapidly as possible given a drive level and source impedance. This requirement is different from that of pulse sharpening and induction accelerator applications. To select the ferrite which best meets kicker magnet criteria, samples of a standard size were obtained from four manufacturers. These samples were subject to pulsed tests where the induced voltage and current were measured at a drive level of $6 \mathrm{kV}$ with a $50 \Omega$ source.

The concept of amplitude independent permeability is only valid at very low induction levels under steady state conditions. Permeability is a function of flux density and frequency. It is straight forward to generate B vs. H curves for a given waveform, frequency and amplitude. However, the application of this information to pulse excitation has several problems. A high voltage pulse testing fixture was constructed to escape these difficulties. So that these test results can be reasonably applied to the design magnet, the samples are approximately the same size and magnetic path length as one pole piece of the actual magnet.

\section{Experimental Apparatus}

There were several iterations on the test fixture and $\mathrm{dB} / \mathrm{dt}$ measuring loop that improved performance. Standard high voltage cable, RG/U220, with a $50 \Omega$ characteristic impedance is used as a pulse forming line. This PFL is split in the middle and the sample stand is inserted. One free end of cable is terminated with an EG\&G GP-86 spark gap and a $50 \Omega$ series resistor to produce a matched source. The source resistance also absorbs reflections from the sample. The other free end of the cable is attached through $10 \mathrm{M} \Omega$. with ferrite beads over the resistors, to the high voltage power supply. The test fixture provides a controlled $\sim 50 \Omega$ impedance up to the sample by means of a tapered coaxial line. The sample region has a center conductor diameter of $5.1 \mathrm{~cm}$ and an outer conductor diameter of $11.4 \mathrm{~cm}$. For quantitative analysis of the response, it is important to provide a controlled and matched impedance to the sample. The test fixture is capable of operating up to approximately $14 \mathrm{kV}$ in air and the spark gap is designed for use between $6 \mathrm{kV}$ and $15 \mathrm{kV}$. The test samples are toroidal with a rectangular cross section. They have a $10.2 \mathrm{~cm}$ outer diameter, a $6.4 \mathrm{~cm}$ inner diameter and are $2.5 \mathrm{~cm}$ deep.

A detailed schematic of the test sample area is shown in Fig. 1. The current is measured in the outside conductor of the coaxial test fixture with a current viewing resistor. This $0.50 \Omega$ resistance is constructed of sixty $30.1 \Omega, \pm 1 \%$ metal film resistors arranged in a continuous band around the outside of the sample area. A $50 \Omega$ source resistance is placed between the current viewing resistance and the $50 \Omega$ hard line to the oscilloscope. The $\mathrm{dB} / \mathrm{dt}$ loop is connected to a $5 \mathrm{k} \Omega$ input impedance $200: 1$ high voltage divider that has a step response time of $\sim 2 \mathrm{~ns}$. The output of the divider is a $50 \Omega$ connection with hard line to the oscilloscope. Ferrite beads are placed around each hard line and both signals are referenced to the same side of the current viewing resistor to reduce ground problems. Delays through the cables and attenuators are identical within $\pm 1 \mathrm{~ns}$.
Current Viewing Resistor 60 Parallel $30.1 \Omega$ Resistors

Equally Spaced Around Perimeter

200:1 High Voltage Divider $5 \mathrm{k} \Omega$ Input Impedance

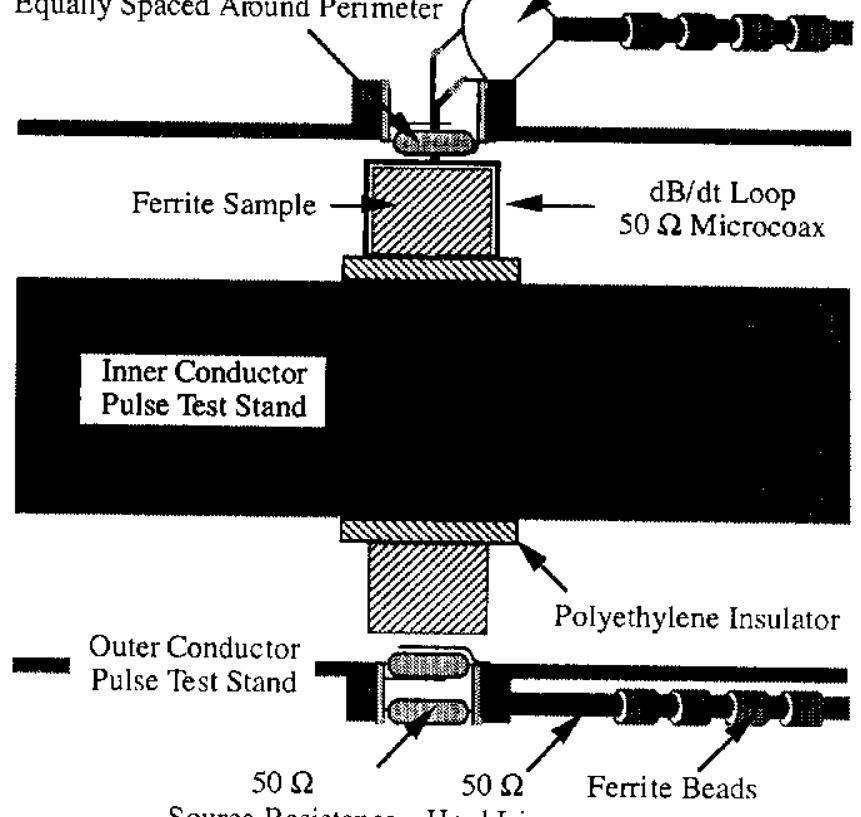

Source Resistance Hard Line

Figure 1

Detail of Sample Test Area

The coaxial line is charged to $12 \mathrm{kV}$ to start a test. The spark gap is triggered and a pulse wave front propagates down the line with an amplitude of $-6 \mathrm{kV}$. This wave is incident onto the test sample. Part of the wave continues through the sample and the rest is reflected back to the source, the spark gap, where it is absorbed by the source resistance. When the wave reaches the charging supply, it is reflected by the open circuit on the cable and comes back to the test fixture. Since the system has reached a stable state between the initial pulse and the pulse reflected from the charging power supply, the line is substantially discharged after one pass. Next, the high voltage supply is turned off, a $60 \mathrm{~Hz}$ source is connected, and the spark gap is shorted to ground. This supply resets the ferrite sample to an unmagnetized initial state. The system was also pulsed repetitively, every several minutes, without resetting the sample to determine the size of the remnant flux density.

The data was digitized by a two channel, $1 \mathrm{GS} / \mathrm{s}$ per channel oscilloscope with a $400 \mathrm{MHz} \mathrm{CW}$ bandwidth and 6 bits of vertical resolution. It was then transferred to a computer for data reduction. The trace pairs were shifted in time until the leading and trailing edges of the current waveforms of the various trials were aligned. Of the 5 to 6 trials for each material, typically 3 or more trials were similar and were used as the basis for comparison. Variations from one trial to the next were primarily due to changes in the switching time of the spark gap, but were also due to changes in the charged line voltage.

Samples were solicited from four companies. Three of these materials were specifically recommended for particle accelerator applications: CMD5005 by Ceramic Magnetics, Inc., 8C11 by Phillips Components, PE12C by TDK. A "reference" material, $4 \mathrm{M} 2$ from Phillips Components, was also tested since many kicker magnets currently operating at Fermilab use a similar material. The CN20 material from Ceramic Magnetics is primarily aimed at EMI suppression and RF transformer applications. All these samples were nickel zinc ferrites. A lithium zinc ferrite was also examined; TT714800 from Trans-Tech. Inc. is primarily used for microwave devices.

The low level sinusoidal permeability and permittivity were also measured for the CMDS005. The s parameters were measured in a $7 \mathrm{~mm}$ diameter airline with a network analyzer. A method similar to the Nicholson-Ross method was used to calculate $\mu$ and $\varepsilon$. 


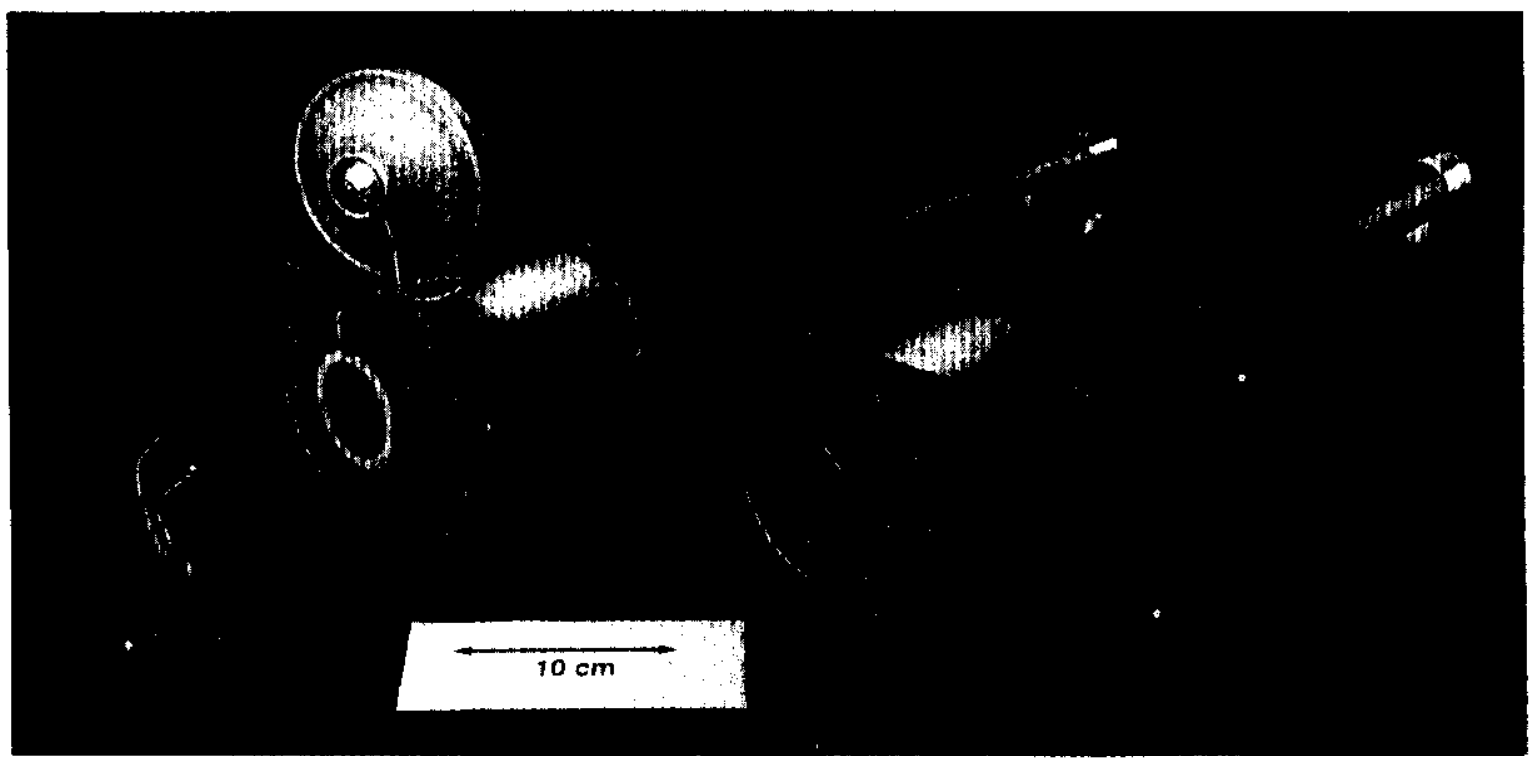

Figure 2, Photograph showing a ferrite sample in the test fixture and the dielectric sample

\section{$\underline{\text { Results }}$}

The operation of the test system was verified by inserting a sample with the same dimensions as the ferrite, but with $\mu_{\mathrm{r}} \approx 1, \varepsilon_{\mathrm{r}} \approx 4$. The area of the $\mathrm{dB} / \mathrm{dt}$ loop was measured and several trials were performed. The response of the $\mathrm{dB} / \mathrm{dt}$ loop showed large oscillations with a period of approximately $8 \mathrm{~ns}$. These oscillations were only seen in the flux density, not the field strength (Fig. 3). The amplitude of the oscillations was not consistent from trial to trial, however the frequency was consistent. This oscillation was not due to a transit time in the $\mathrm{dB} / \mathrm{dt}$ sense loop or propagation times through the sample. These would happen in less than 1 ns. While the exact cause of the oscillation is not known, it may be due to discontinuities in the test fixture, to higher order modes in the test fixture, or to both. The first higher order mode, $T E_{11}$, has a cutoff frequency of $1.16 \mathrm{GHz}$ in the unloaded line and the transit time from the sample to the end of the taper, $47 \mathrm{~cm}$, is approximately $1.6 \mathrm{~ns}$. In any case, the integration of $\mathrm{d} B / \mathrm{d}$ to give $\mathrm{B}$ was relatively consistent and the oscillations were not present with a ferrite sample.

Calculation of the relative permeability from Fig. 3 gives a value of $0.92 \pm 0.07$. However, the relative accuracy between samples due to measurement uncertainty is $\pm 2 \%$. The rise time of the applied pulse is approximately $15 \mathrm{~ns}$.

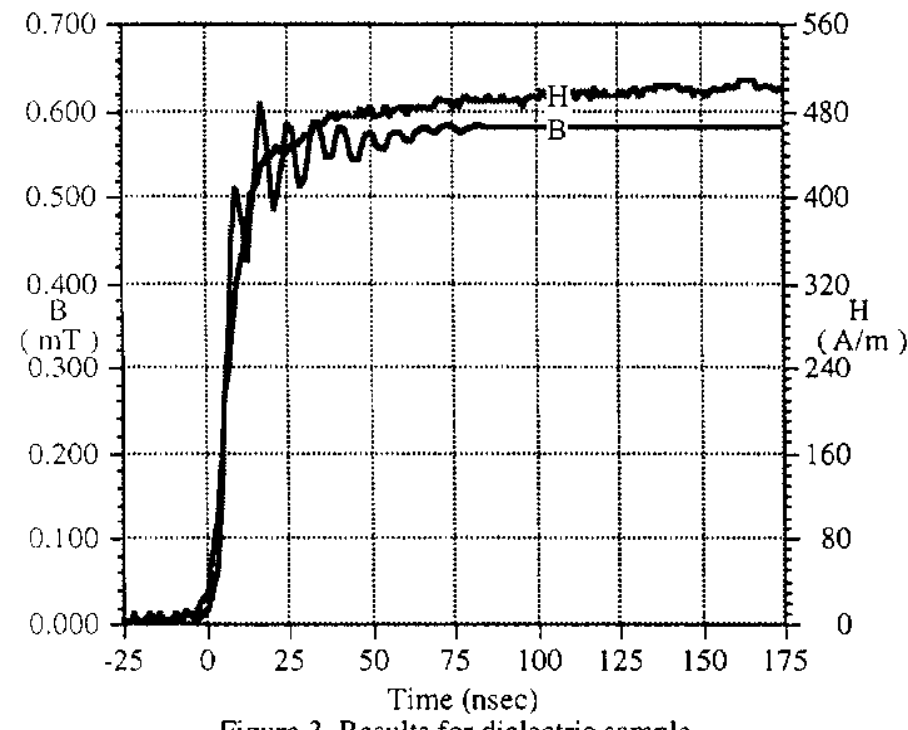

The flux density and field strength for ferrite samples starting at both an unmagnetized state and a remnant point are shown in Fig 4. There are several points to note about this data. First, the field values are at the geometric mean radius of the sample assuming the fields fall inversely with distance. Next, the ferrite is driven into saturation in all cases due to a high drive level and the absence of an air gap. Also, it should be noted that magnetic properties for ferrites can vary by as much as $\pm 20 \%$ from batch to batch for the same material from the same manufacturer. Differences much less than this have little relevance in determining which material to use. Finally, the data is presented in a field $v s$. time format instead of a field $v s$. field format. This more clearly shows the different time regimes during the pulse.

As a follow up to the pulse data, the low level sinusoidal characteristics of CMD5005 were investigated. Two different network analyzers (HP 8753C and HP 8510) and a $7 \mathrm{~mm}$ coaxial air line were used to measure the full four port $s$ parameters. From these, and knowing the length and position of the sample in the air line, the complex permeability and permittivity of the material can be calculated. At the lower frequencies, the same samples and air line were used and the capacitance and inductance measured (HP 4194A). From these measurements, the real $\varepsilon_{r}$ and $\mu_{r}$ can be calculated. Figs. 5 and 6 show real permeability and permittivity over several decades. While error bars are not shown, they are substantial for frequencies much less than $10 \mathrm{MHz}, \sim 100$ for $\mu_{\mathrm{r}}$ and $\sim 1$ for $\varepsilon_{\mathrm{r}}$, but smaller for frequencies much greater than $10 \mathrm{MHz}, \sim 0.1$ for $\mu_{\mathrm{r}}$ and $\varepsilon_{\mathrm{r}}$.

\section{Discussion}

For this application spatial and temporal variations in the air gap flux density must be less than $1 \%$. Also the ferrite losses must be low such that the magnetization is stable in the shortest possible time. The selection of the "best" ferrite is therefore based on these criteria:

- fastest time to a given flux density

- low remnant flux density

- high saturation magnetization

How well these requirements are met is determined by examining Fig. 4. There are at least three time regimes shown: a transit time through the ferrite, energy transfer to the ferrite, and finally ferrite saturation. The transit time and energy transfer are directly related to the fast flux density rise time and low remnant flux density. The fast flux density rise time and low remnant flux densily are also interrelated through the loss mechanism.

There are several mechanisms limiting the rise time: the spin relaxation of the ferrite crystal, available peak power given a fixed source impedance, the inherent mismatch between the ferrite section and the pulse forming line (PFL), and the magnetization energy loss. The ferrite spin relaxation does not directly appear to be a limiting factor in this case. And since in the application the inductance is 
dominated by the air gap, the $L /\left(2 Z_{0}\right)$ rise time will be the same for each material. However, the impedance mismatch and energy loss need to be examined further.

The delay between the field strength rise and the flux density rise is designated as the transit time regime. Experimentally, there is a very small increasing flux density during this initial time. The reason for this delay is that the ferrite loaded section of the coaxial line initially has a high wave impedance compared to the $50 \Omega$ system and most of the incident wave is initially reflected. Until the transmitted wave travels through the ferrite, reflects from the back surface and returns to the front surface, the ferrite presents a high wave impedance.

With a simple dielectric there is a delay of less than $2 \mathrm{~ns}$, Fig. 3, while the delay with the ferrite is 5 to $10 \mathrm{~ns}$. This delay increases with the total change in flux density, i.e. the delay is shorter for the same material when the repetitive flux density swing is less than the initial flux density swing. This implies the delay depends on the internal state of the material. If the delay is a round trip propagation time through the ferrite, then the group velocity is $510^{6}$ to $10^{7} \mathrm{~m} / \mathrm{s}$. If this delay was simply due to a $\mathrm{EM}$ wave, given $\varepsilon_{\mathrm{r}} \approx 12$ the relative permeability during this initial period is between 70 and 300. For comparison, the $4 \mathrm{M} 2$ has a delay of $5 \mathrm{~ns}$ and manufacturer's $\mu_{i}=140$ while the CMD5005 has a delay of $8 \mathrm{~ns}$ and manufacturer's $\mu_{\mathrm{i}} \approx 1600$. Since the cause of the delay has not been definitely determined, the coincidence of the $\sim 8 \mathrm{~ns}$ oscillation and the $\sim 8 \mathrm{~ns}$ delay is disconcerting.

The energy loss during magnetization is related to the remnant flux density. The remnant flux density ranges from $\sim 0 \%$ to $\sim 90 \%$ of the saturation flux density for the materials tested. Theoretically energy can only be supplied to the ferrite during the pulse. All the energy required to demagnetize the sample is supplied from energy stored by the various internal mechanisms of the ferrite. Since the resistivity of the accelerator materials is generally high, $\rho>10^{5} \Omega \mathrm{cm}$, and the dielectric losses are generally small, a low remnant induction with single polarity pulse excitation in a matched system implies that the magnetization process has lower losses. The magnetization loss is due primarily to either domain rotation or domain wall motion.

When total reversal of the ferrite flux was studied, domain wall motion limited by spin relaxation was assumed as the mechanism for magnetization [1] and spin relaxation damping was assumed as the main loss mechanism. For that application the material is initially saturated in the opposite sense and the saturation level of the material is the limiting factor.

Snoek [2] determined that if initial permeability is due to the rotation of domains and the domains are assumed to be ellipsoidal, the saturation magnetization can be related to the frequency of maximum magnetic loss by

$$
f_{\max }=\frac{\gamma M_{s a t}}{3 \pi\left(\mu_{i}-1\right)}
$$

Substituting for the gyromagnetic ratio, $\gamma$, of $0.2210^{6}(\mathrm{rad} / \mathrm{s}) /(\mathrm{A} / \mathrm{m})$ and the measured CMD5005 values: an initial permeability, $\mu_{i}$, of 1600 , a saturation magnetization, $M_{\mathrm{sat}}$, of $0.33 / \mu_{0} \mathrm{~A} / \mathrm{m}$, the peak loss occurs at $\mathrm{f}_{\max }$ of $3.8 \mathrm{MHz}$. This is near the middle of the transition region of calculated $\mu$, Fig. 5, which is where the imaginary part of $\mu$ should be largest. Unfortunately, for samples commonly used, the complex permeability is difficult to measure in this frequency range. The sample size is approaching fractions of a wavelength so the lump component model is not valid but the phase shift is less than can be accurately measured with the s parameter test set. The dominant loss mechanism in this application does not seem to be determinable from the test measurements made.

Finally, the higher the saturation flux density, the greater the margin for use at a given flux density or the higher the flux density in the ferrite. This is only useful, in this application, if the losses are low. The flux density flat top is designated as the saturation regime. Even though $\varepsilon_{\mathrm{r}}$ and $\mu_{\mathrm{r}}$ are still the order of 10 , the fields have almost reached a steady state and therefore the reflected waves are small. The measured saturation levels agree with the manufacturers' published $1 \mathrm{kHz}$ hysteresis data within $\pm 15 \%$, except for TT71-4800 and 4M2.

The $4 \mathrm{M} 2$ measured saturation flux density of $0.18 \mathrm{~T}$ is $40 \%$ lower than the manufacturer's value of $0.25 \mathrm{~T}$. The manufacturer's saturation magnetization for the TT71-4800 is $0.48 \mathrm{~T}$; much closer to the measured flux density saturation of $0.47 \mathrm{~T}$ than to the manufacturer's $2 \mathrm{kHz}$ hysteresis loop flux density maximum of 0.35 T. The TT71-4800 has the highest saturation flux density, however the magnetization losses are also relatively high. This ferrite is typically operated only near saturation in millimeter wave applications.

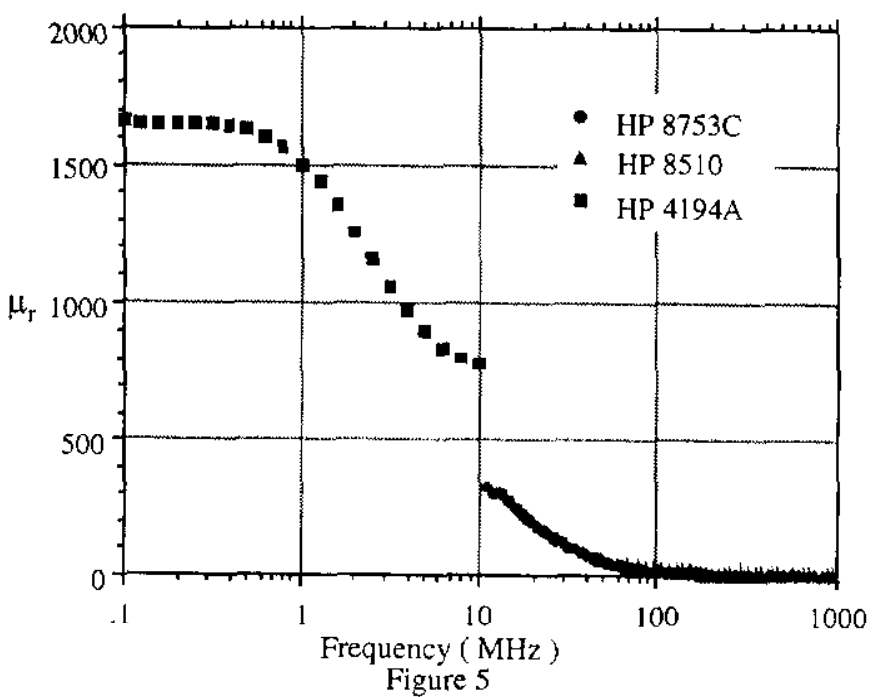

Real Permeability vs. Frequency

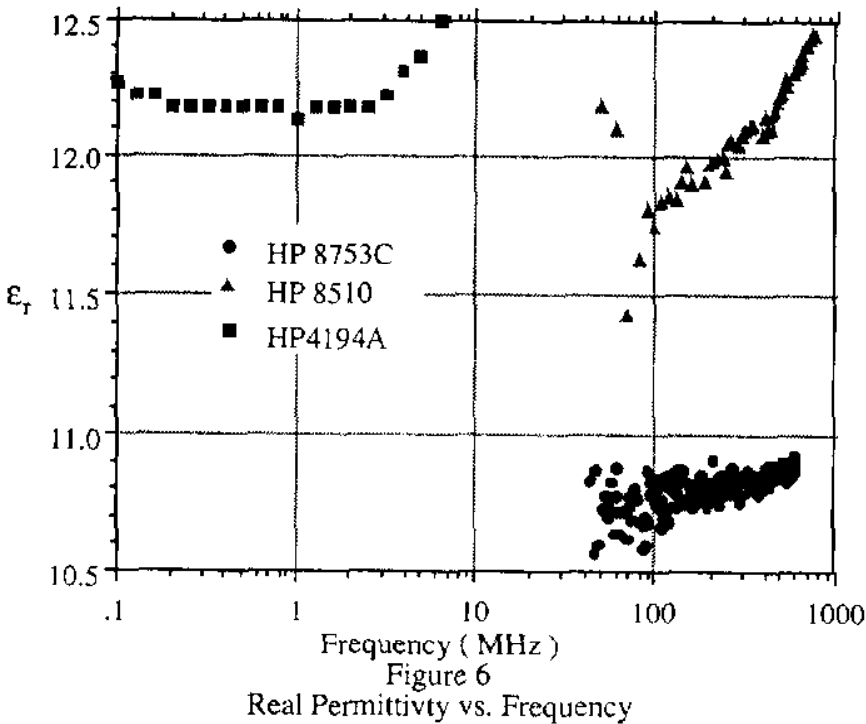

\section{Conclusions}

A pulse test stand was constructed and the pulse response of six different materials was examined. The $8 \mathrm{C} 11$ definitely has the lowest losses for a substantial saturation flux density. The CMD5005 has the highest saturation flux density for slightly higher losses than $8 \mathrm{C} 11$. Also, the flux density for this application is $0.15 \mathrm{~T}$ and that is within even the repetitive flux density excursion of these materials. The PE12C has slightly higher losses than CMD5005 and slightly higher saturation than $8 \mathrm{C} 11$. The $4 \mathrm{M} 2$, typically used for pulse transformers, has a fairly low saturation flux density but also very low loss. The $\mathrm{CN} 20$ seems similar to the $8 \mathrm{C} 11$, but with higher losses. Either the 8C11 or CMD5005 material is appropriate for this application. The CMD5005 might have an advantage in higher field applications, while the $8 \mathrm{C} 11 \mathrm{might}$ have an advantage in high field high repetition rate applications due to lower losses. The CMD5005 material was chosen for this application for a combination of reasons. The project has advanced past the theoretical stage and measurements on the actual magnet are scheduled to begin shortly [3].

\section{References}

[1] Avery, R.K., ef al, A simple domain model of flux reversat in ferrites, Journal Applied Physics, Vol. 67, No 9, May 11990

[2] Snoek, J.L., Dispersion and atsorption in magnetic ferrites at frequencies above $1 \mathrm{Mc} / \mathrm{s}$, Physica Amsterdam, Vol. 14, 1948

[3] Dinkel, J.A, Jensen, C.C., A precise high field injection kicker magnet for the Fermilab Tevatron, 9th IEEE Pulse Power Conf. 


\section{Comparison of Dielectric and CMD5005}

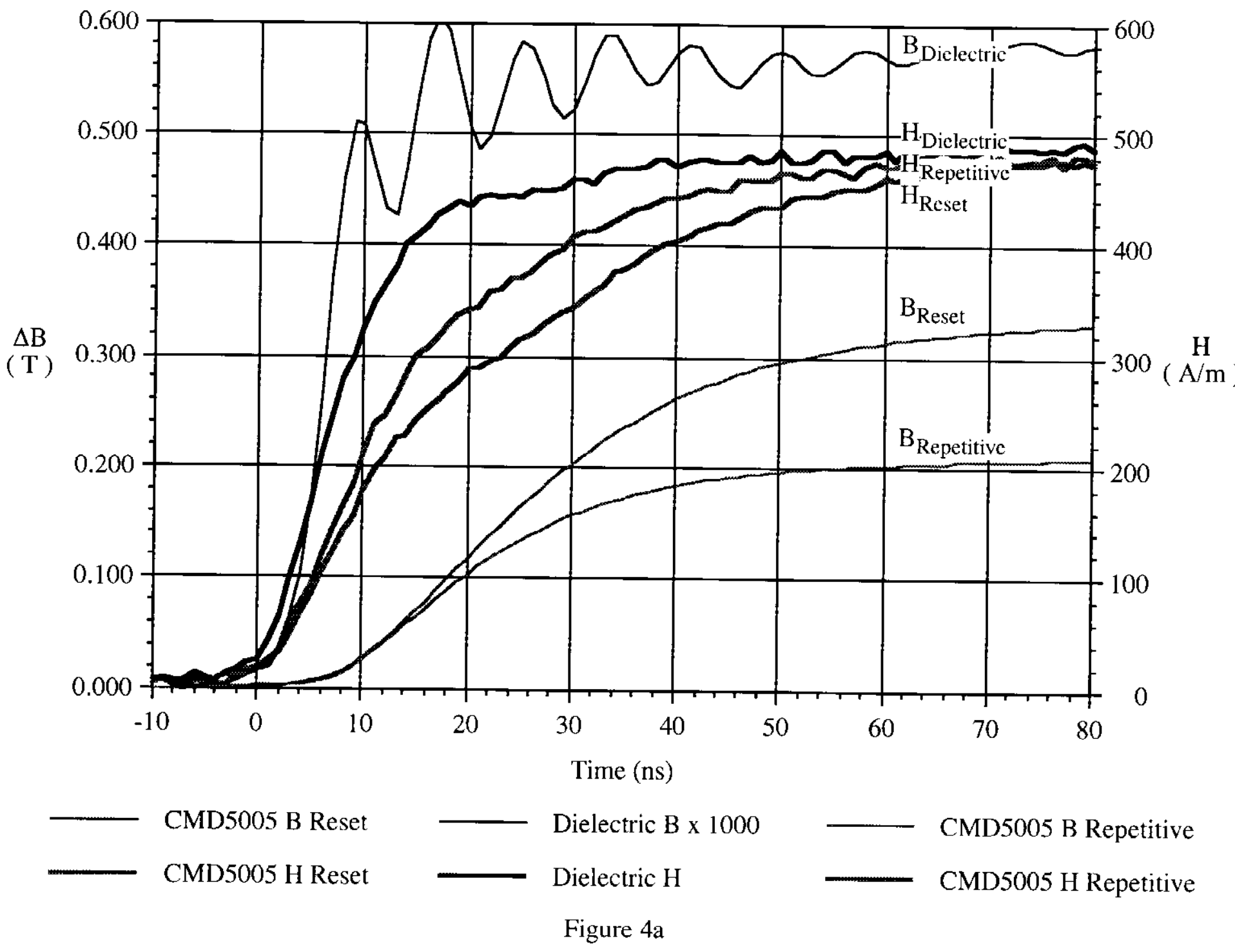




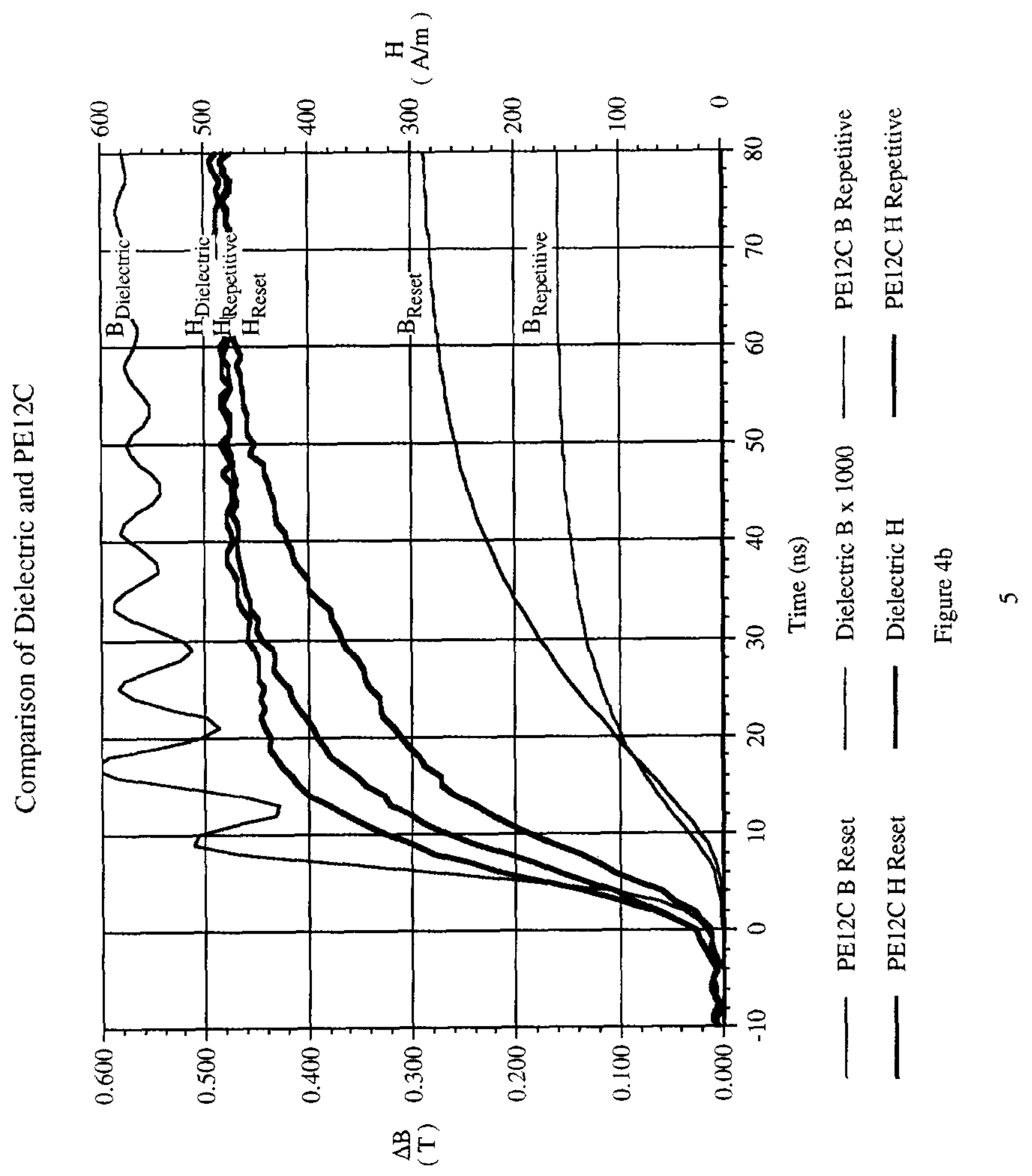




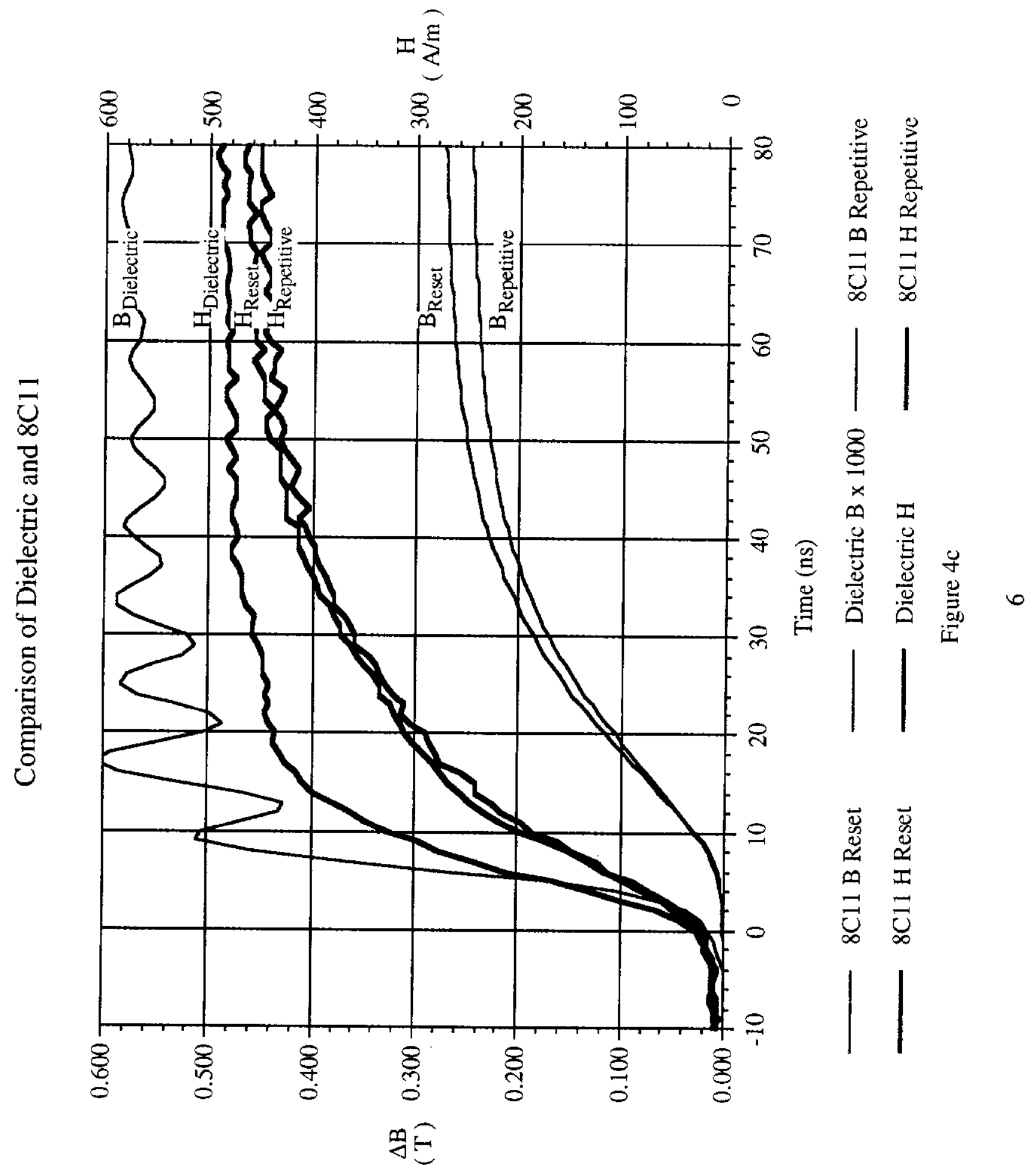




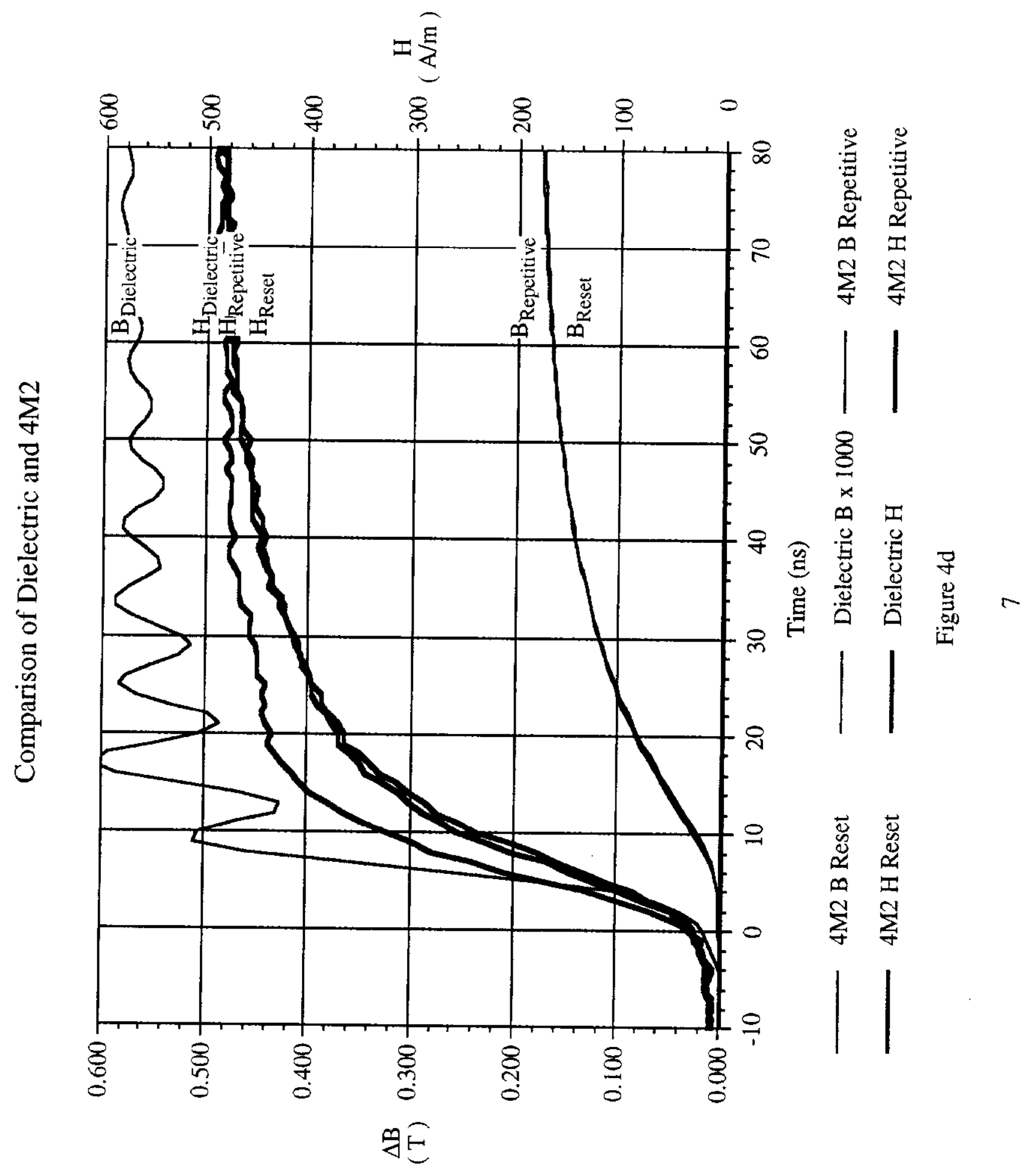


Comparison of Dielectric and $\mathrm{CN} 20$

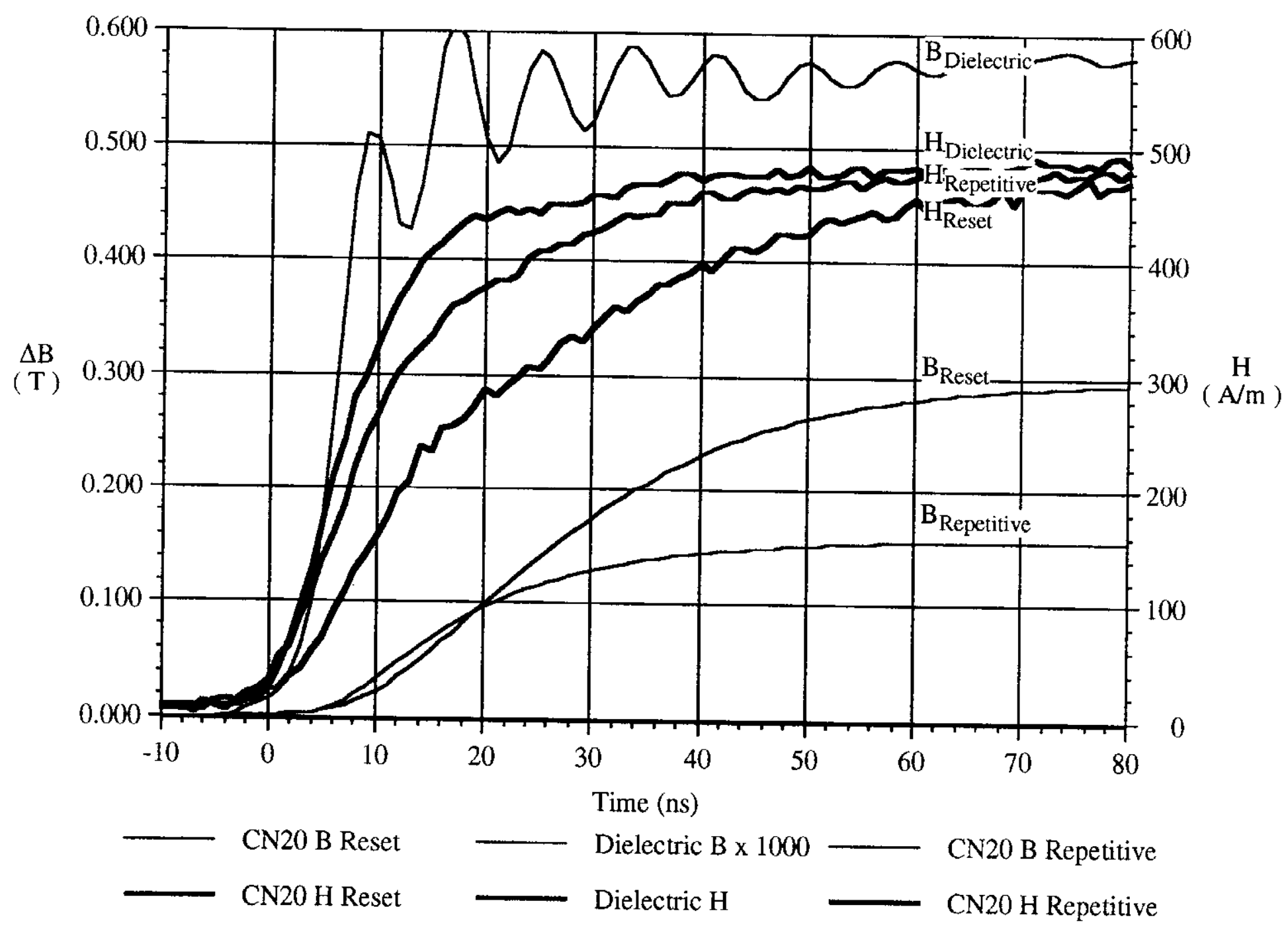

Figure 4e 


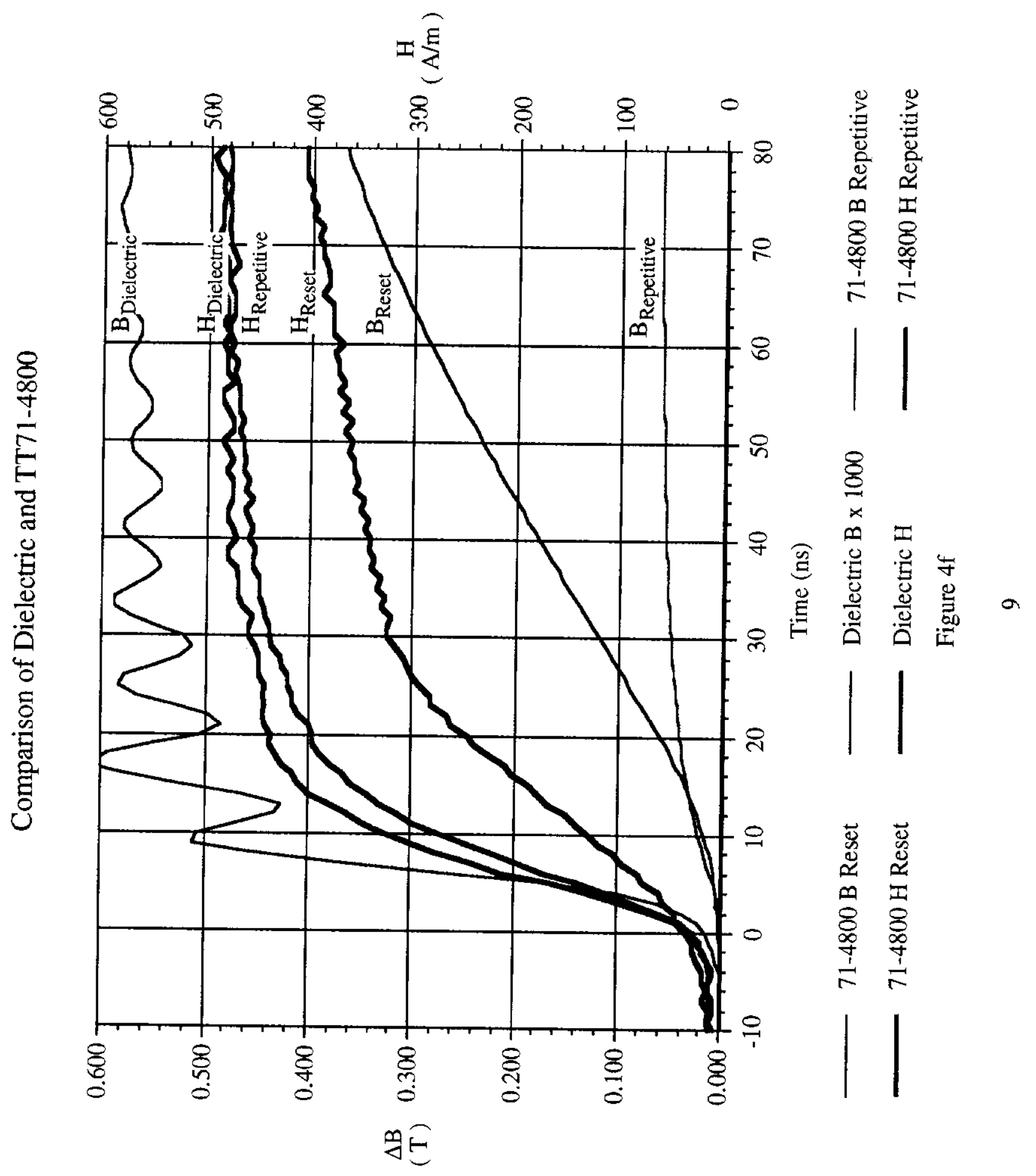

